\title{
Impact of Carbon Support Corrosion on Performance Losses in Polymer Electrolyte Membrane Fuel Cells
}

\author{
Friedemann Hegge, ${ }^{1,2,3}$ Jonathan Sharman, ${ }^{4}$ Riko Moroni, ${ }^{1}$ Simon Thiele, ${ }^{2,3}$ \\ Roland Zengerle,, 5 Matthias Breitwieser, 1,5 and Severin Vierrath $\oplus^{1,5,6, *, z}$ \\ ${ }^{1}$ Laboratory for MEMS Applications, IMTEK Department of Microsystems Engineering, University of Freiburg, \\ 79110 Freiburg, Germany \\ ${ }^{2}$ Forschungszentrum Jülich GmbH, Helmholtz-Institute Erlangen-Nürnberg for Renewable Energy (IEK-11), \\ Forschungszentrum Jülich, 91058 Erlangen, Germany \\ ${ }^{3}$ Department of Chemical and Biological Engineering, Friedrich-Alexander-Universität Erlangen-Nürnberg, \\ 91058 Erlangen, Germany \\ ${ }^{4}$ Johnson Matthey Technology Centre, Reading RG4 9NH, United Kingdom \\ ${ }^{5}$ Hahn-Schickard, 79110 Freiburg, Germany \\ ${ }^{6}$ FIT - Freiburg Center for Interactive Materials and Bioinspired Technologies, 79110 Freiburg, Germany
}

\begin{abstract}
Corrosion of the carbon support leads to a severe decay in the performance of PEM fuel cells, mainly due to an increase in the oxygen transport resistance. To investigate the effect of degradation on oxygen transport, we cycled MEAs between 1-1.5 V and analyzed the electrode structure with FIB-SEM tomography at various ageing states. The tomography results show that the electrode structure changes over 1000 cycles in terms of thickness $(7.8$ to $6.5 \mu \mathrm{m})$, porosity (44 to $38 \%)$ and diffusivity $\left(9\right.$ to $\left.8 \cdot 10^{5} \mathrm{~m}^{2} \mathrm{~s}^{-1}\right)$. Limiting current measurements in the wet (hydrogen/air) and dry state (hydrogen pumping) allowed the pressure dependent and pressure independent mass transport resistances to be distinguished and to quantify the impact of product water. The pressure independent resistance increased from 24 to $41 \mathrm{sm}^{-1}$. Considering the marginal contribution of the catalyst pore space resistance $\left(3\right.$ to $\left.4 \mathrm{sm}^{-1}\right)$ it is concluded that the largest portion of the increase (50\%) is caused by an increased local mass transport resistance. This is due to a decrease of the electrode roughness factor (282 to 169). The limiting current under wet conditions shows that another $44 \%$ could stem from a change in the wetting behavior, while $6 \%$ remains unexplained.

(C) The Author(s) 2019. Published by ECS. This is an open access article distributed under the terms of the Creative Commons Attribution 4.0 License (CC BY, http://creativecommons.org/licenses/by/4.0/), which permits unrestricted reuse of the work in any medium, provided the original work is properly cited. [DOI: 10.1149/2.0611913jes]

Manuscript submitted June 7, 2019; revised manuscript received August 6, 2019. Published August 21, 2019.

Carbon corrosion of the catalyst support can severely decrease the performance of polymer electrolyte membrane fuel cells (PEMFCs). The corrosion can occur during normal operation, since it is only metastable above the standard electrode potential of carbon of $0.207 \mathrm{~V}$ vs. the reversible hydrogen electrode, but is strongly enhanced by high potentials at the anode during cell reversal and at the cathode during start-up or shutdown. ${ }^{1}$ The high potential difference during start-up and shutdown is caused by an oxygen front in the hydrogen at the anode side as originally found by Reiser et al. $^{2}$ The carbon corrosion, especially in the cathode, leads to a severe loss of performance. There are three common hypotheses for the performance loss related to carbon corrosion. Firstly, it is supposed that the catalyst layer (CL) porous structure collapses due to the loss of carbon support, decreasing the diffusivity in the pore space and thus increasing the mass transport losses. ${ }^{3}$ Secondly, detachment and agglomeration of catalyst particles lead to a loss of electrochemically active surface area (ECSA) which is commonly linked to an increase of kinetic losses. Thirdly, it is assumed, that carbon corrosion involves the formation of oxygen surface groups which are hydrophilic. This loss of hydrophobicity might lead to an increased liquid water uptake in the CL leading to increased mass transport losses. . $^{1,4}$

Even though carbon corrosion phenomena are well recognized, the associated performance degradation has rarely been quantitatively investigated. This quantification is crucial though, in order to understand which degradation phenomenon needs to be mitigated, to develop more stable PEMFCs. There have been several studies which investigated electrochemical performance degradation and the physical changes in degraded cathodes by X-Ray (nano CT), ${ }^{5}$ focused ion beam - scanning electron microscope (FIB-SEM) ${ }^{3,6,7}$ or transmission electron microscopy. ${ }^{8-10}$ Among the existing publications, there are a few, which link the performance degradation to physical changes of the CL by tomography or electrochemical measurements. In a FIB-SEM study Schulenburg et al. ${ }^{3}$ observe a loss of porosity of the cathode catalyst layer of a PEMFC following a major loss in performance. The

*Electrochemical Society Student Member

${ }^{z}$ E-mail: severin.vierrath@imtek.de porosity decreased to a level at which the increased diffusion resistance through the pore space became the dominant loss mechanism. The degree of degradation captured in their study was however, already too high to be relevant for operation in a fuel cell vehicle (losses greater than $200 \mathrm{mV}$ at $1.5 \mathrm{~A} / \mathrm{cm}^{2}$ in $\mathrm{H}_{2} / \mathrm{O}_{2}$ operation at 2.5 bar abs and $80^{\circ} \mathrm{C}$ and a decrease of CL porosity to approx. $5 \%$ ). Another FIBSEM investigation by Star et al. ${ }^{7}$ comes to the conclusion, that the carbon support structure is first hollowed, i.e. the porosity increases, and then the structure collapses, as concluded by a subsequent decrease of porosity. Remarkably, they observe a significant loss of performance before a decrease in porosity and diffusivity of the CL. Nevertheless, Star et al. do not quantitatively assign the physical changes of the PEMFC cathode to the performance losses.

In the present work, performance loss related to carbon support corrosion is investigated via both tomography and electrochemical measurements. With the help of the tomographic data and electrochemical measurements, the individual fractions of the degradation mechanisms are quantified, revealing the main reasons for the performance loss.

\section{Methods and Experimental}

Accelerated stress tests.-Accelerated stress testing was conducted on membrane electrode assemblies (MEAs) with a cell area of $1 \mathrm{~cm}^{2}$, providing differential conditions, to ensure all parts of the active area experienced the same conditions. For MEA preparation, catalyst layers were laminated onto Nafion NRE 211 membranes. The cathode loading was $0.34 \mathrm{mg}_{\mathrm{Pt}} \mathrm{cm}^{-2}$, the $\mathrm{Pt} / \mathrm{C}$ ratio was 1 and the ionomer to carbon weight ratio was 0.8 , with a total ionomer content of $28.6 \mathrm{wt} \%$. Sigracet $29 \mathrm{BC}$ gas diffusion layers (GDLs) with a microporous layer (MPL) were applied on cathode and anode side. A series of samples was produced for accelerated stress testing following the DOE catalyst support cycling protocol, ${ }^{11}$ which cycles the voltage between 1.0 and $1.5 \mathrm{~V}$ at $500 \mathrm{mV} \mathrm{s}^{-1}$ in a $\mathrm{H}_{2} / \mathrm{N}_{2}$ configuration at $80^{\circ} \mathrm{C}$ and $100 \%$ RH. Samples were subjected to 100,500 and 1000 cycles and the performance losses were characterized by measuring polarization curves under air operation. Subsequently changes in the cathode 
microstructure were analyzed by FIB-SEM tomography. In addition, one sample was further investigated by determining the limiting current in a hydrogen pump setup and measuring the active surface area by cyclic voltammetry after the respective cycle numbers. 1000 cycles was chosen as a maximum number of degradation cycles, as the voltage loss observed at that point significantly exceeded the limit given by the DOE of $30 \mathrm{mV}$ at $1.5 \mathrm{~A} / \mathrm{cm}^{2}$ at $80^{\circ} \mathrm{C}, 80 \% \mathrm{RH}, 1.5 \mathrm{bar}_{\mathrm{abs}}$.

Structural investigations. - The structural changes of the catalyst support were investigated by reconstructing a cubic volume of $3 \mu \mathrm{m}$ $\times 3.9 \mu \mathrm{m} \times 1.2 \mu \mathrm{m}$ of samples from each ageing stage mentioned above (Figure 3). For this, a FEI Scios 2 FIB-SEM was used. FIB cutting was performed at a current of $300 \mathrm{pA}$ and a cutting distance of $9 \mathrm{~nm}$. The SEM images were recorded at an acquisition angle of $52^{\circ}$, an acceleration voltage of $5 \mathrm{kV}$ and a pixel size of $3 \mathrm{~nm}$. The process of obtaining the $3 \mathrm{D}$-representation of the microstructure involved acquisition, geometrical alignments and segmentation and was implemented in Matlab. In order to enhance the contrast between pores and solid material the samples were infiltrated via atomic layer deposition of $\mathrm{ZnO}$ as introduced in previous work. ${ }^{12}$ With the $3 \mathrm{D}$ reconstruction, morphological and transport parameters were calculated. The porosity was calculated by summarizing the number of pore voxels in the tomographic image and dividing that value by the overall number of voxels. The diffusivity was calculated with the DiffuDict module of GeoDict. The pore sizes of the catalyst layer $\left(\mathrm{D}_{50}\right.$ between $77 \mu \mathrm{m}$ and $81 \mu \mathrm{m}$ corresponding to a Knudsen number of 0.7 to 0.8 ) are in the range of the mean free path length of the diffusing oxygen and water molecules. Thus, the diffusive gas transport is governed by interparticle collisions (Laplace diffusion) and particle wall collisions (Knudsen diffusion) in a similar amount. Therefore, the diffusion coefficient was calculated by the Bosanquet approximation. ${ }^{13}$

$$
D_{\text {Bosanquet }}=\frac{1}{\frac{1}{D_{\text {Knudsen }}}+\frac{1}{D_{\text {Laplace }}}}
$$

Here, $D_{\text {Knudsen }}$ is the Knudsen Diffusivity and $D_{\text {Laplace }}$ is the Laplace Diffusivity. Taking the reciprocal value and multiplying with the layer thickness, the Bosanquet approximation calculates the diffusion resistance as the sum of Laplace and Knudsen diffusion resistance. Bulk diffusion coefficients were calculated for $1.5 \mathrm{bar}_{\mathrm{abs}}$ and $80^{\circ} \mathrm{C}$ with a mean free path length of $58 \mathrm{~nm}$ and a mean thermal velocity of $483 \mathrm{~m} \mathrm{~s}^{-1}$ assuming unimolecular diffusion. ${ }^{14}$ The cathode layer thickness was determined by averaging the measured thickness at four spots on each sample. For this, at each measuring spot, a trench of $6 \mu \mathrm{m} \times 8 \mu \mathrm{m} \times$ $10 \mu \mathrm{m}$ was cut into the catalyst layer using the FIB. Subsequently the CL thickness was measured by SEM.

Electrochemical measurements.-All electrochemical measurements were performed in a $5 \mathrm{~cm}^{2}$ single cell with serpentine flow field using a Scribner $850 \mathrm{e}$ fuel cell testing system. The active area of the cell was reduced to $1 \mathrm{~cm}^{2}$ in order to realize a small pressure drop at significantly over-stoichiometric flows ( $>880$ for hydrogen pumping and $>11$ in $\mathrm{H}_{2}$ /air operation) and thereby differential conditions. Polarization (I-V) curves were recorded under $\mathrm{H}_{2}$ /air with flows of $0.2 / 0.5 \mathrm{slpm}$ at $80^{\circ} \mathrm{C}, 80 \%$ relative humidity and an absolute pressure of 1.5 bars (in accordance with the DOE protocol). The cell voltage $E_{\text {cell }}$ was divided into individual contributing portions according to Suermann et al. ${ }^{15}$

$$
E_{\text {cell }}=E_{\text {rev }}-\eta_{\text {kin }}-\eta_{H F R}-\eta_{M T R, \text { prot } C L}
$$

where $E_{\text {rev }}$ is the reversible cell voltage calculated at operating temperature and pressure by the temperature dependent equilibrium potential ${ }^{16} E_{0}$ and the Nernst equation

$$
E_{\text {rev }}=E_{0}+\frac{R T}{2 F} \ln \left[\frac{a_{\mathrm{H}_{2} \mathrm{O}}}{a_{\mathrm{H}_{2}} a_{\mathrm{O}_{2}}{ }^{1 / 2}}\right]
$$

where $R$ is the universal gas constant, $T$ the temperature, $\mathrm{F}$ the Faraday constant and $a_{\mathrm{H}_{2} \mathrm{O}}, a_{\mathrm{H}_{2}}$ and $a_{\mathrm{O}_{2}}$ are the activities of water, hydrogen and oxygen.
The kinetic overpotential was calculated as the difference between the reversible cell voltage and the extrapolated Tafel fit of the high frequency resistance (HFR) free cell voltage

$$
\eta_{\text {kin }}=E_{\text {rev }}-(a+b \log (i))
$$

with $i$ being the current density and $a$ and $b$ the Tafel constants, obtained from a fit in the kinetic current density region between 10 and $100 \mathrm{~mA}$ $\mathrm{cm}^{-2}$, where the mass transport overpotential can be neglected. The HFR overpotential $\eta_{H F R}$ was measured and the remaining difference between the HFR free voltage and the kinetic corrected voltage (the extrapolated Tafel fit) yields the sum of mass transport overpotential and protonic transport resistance in the CL, denoted by $\eta_{M T R, p r o t} C_{L}$.

The electrochemically active surface area (ECSA) was measured by cyclic voltammetry $(\mathrm{CV})$. The $\mathrm{CV}$ was conducted under a $\mathrm{H}_{2} / \mathrm{N}_{2}$ atmosphere. The cell was stabilized for $60 \mathrm{~min}$ with $\mathrm{H}_{2} / \mathrm{N}_{2}$ flows of $0.2 / 0.2 \mathrm{slpm}$ at $80^{\circ} \mathrm{C}, 100 \%$ relative humidity and ambient pressure (1015 mbar). For the CV measurement, the cathode flow was stopped, whereas the other conditions were maintained. Five cycles were performed between $0.05 \mathrm{~V}$ and $0.8 \mathrm{~V}$ at a scan rate of $20 \mathrm{mVs}^{-1}$. The ECSA was calculated from the CV measurements by averaging the charge of the hydrogen adsorption and desorption peaks of the last four cycles.

Limiting current measurements were performed in two setups, a) in normal $\mathrm{H}_{2}$ /air operation and b) in hydrogen pump mode. The hydrogen pump mode allows determination of the mass transport resistance (MTR) without the influence of water generation in the catalyst layer. Due to the unsaturated conditions it is assumed that liquid water does not exist in the macropores, ${ }^{17,18}$ while the ionomer is humidified by the incoming gases. Hydrogen pumping was conducted according to Spingler et al., ${ }^{19}$ Freiberg et al. ${ }^{20}$ and Schuler et al. ${ }^{21}$ with $0.5 \mathrm{slpm}$ of $0.1 \% \mathrm{H}_{2}$ in $\mathrm{Ar}$ on the working electrode and $0.2 \mathrm{slpm}$ of $2 \% \mathrm{H}_{2}$ in $\mathrm{Ar}$ on the counter electrode. The limiting current was measured at 5,50 and $100 \mathrm{kPa}$ backpressure, $80^{\circ} \mathrm{C}$ and $80 \%$ relative humidity. In $\mathrm{H}_{2}$ /air operation the limiting current was measured at the polarization conditions mentioned above and a voltage of $0.1 \mathrm{~V}$.

\section{Theory}

The mass transport overpotential in PEMFCs is governed by reactant transport to the cathode catalyst sites. It can be calculated based on Fick's law, Faraday's law and the assumption that the reactant concentration at the catalyst is zero at limiting current. ${ }^{22}$ In case of the hydrogen pump setup, the hydrogen transport resistance $R_{H 2}$ is calculated by

$$
R_{H 2}=\frac{2 F C_{H 2, \text { channel }}}{\mathrm{i}_{\text {lim }}}
$$

where $\mathrm{F}$ is the Faraday constant, $C_{H 2 \text {, channel }}$ the average hydrogen concentration at the gas channel and $\mathrm{i}_{\text {lim }}$ the limiting current. $C_{H 2 \text {, channel }}$ is calculated by

$$
C_{H 2, \text { channel }}=\frac{p}{R T} \frac{\dot{n}_{H 2, \text { feed }}-\dot{n}_{H 2, \text { lim }}}{\dot{n}_{\text {feed }, \text { dry }}}\left(1-x_{H 2 O}\right)
$$

with $p$ being the cell pressure $R$ the universal gas constant, $T$ the temperature in K, $\dot{n}_{H 2, \text { feed }}$ the molar hydrogen inlet flux, $\dot{n}_{H 2, \text { lim }}$ the molar flux of consumed hydrogen at limiting current, $\dot{n}_{\text {feed,dry }}$ the molar inlet flow of dry gas and $x_{H 2 O}$ the mole fraction of water in the humidified inlet gas. Hereby the hydrogen flux at limiting current is calculated as:

$$
\dot{n}_{H 2, \lim }=\frac{i_{\text {lim }}}{2 F}
$$

It should be noted that the set cell back pressure does not correspond to the pressure inside the cell. Therefore, the cell pressure and the $\mathrm{RH}$ were corrected considering the measured pressures at the inlet and outlet of the cell.

By varying the pressure, the MTR can be divided into a pressuredependent part $R_{H 2, p}$ (Laplace gas diffusion in pore space) and a pressure-independent part $R_{H 2, p \text { indep }}$ (interfacial resistance, diffusion 


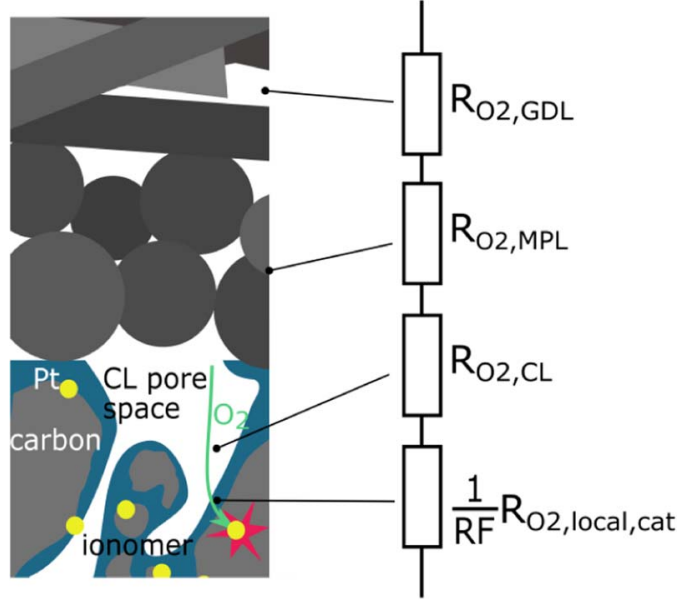

Figure 1. Series connection of oxygen mass transport resistances in a PEMFC comprising the MTR of the GDL $\left(\boldsymbol{R}_{\boldsymbol{O} 2, \boldsymbol{G D L}}\right)$, of the MPL $\left(\boldsymbol{R}_{\boldsymbol{O} 2, \boldsymbol{M P L}}\right)$, of the CL pores $\left(\boldsymbol{R}_{\boldsymbol{O} 2, C L}\right)$ and of the ionomer covering the active catalyst surface including interface resistances $\left(\frac{1}{R F} \boldsymbol{R}_{\boldsymbol{O} 2, \text { Local }, \text { cat }}\right)$.

resistance of the ionomer film covering the platinum catalyst and Knudsen diffusion in the pore space).

$$
R_{H 2}=R_{H 2, p \text { indep }}+R_{H 2, p}
$$

where $R_{H 2, p}$ is linearly correlating with the pressure.

$$
R_{H 2, p}=C p
$$

This linear correlation only holds true if no, or only a negligible amount, of water is produced in the CL. For this reason, hydrogen pump experiments were chosen for determining the pressureindependent and the dry pressure-dependent resistance. In order to calculate the oxygen transport resistance $\mathrm{R}_{\mathrm{O} 2}$ from the hydrogen transport resistance $\mathrm{R}_{\mathrm{H} 2}$ for the pressure-independent MTR (i.e. diffusion resistance of the ionomer and Knudsen diffusion resistance of the pore space), unimolecular diffusion is assumed. ${ }^{20}$ This yields:

$$
R_{O 2, p \text { indep }}=R_{H 2, p \text { indep }} \sqrt{\frac{M_{O 2}}{M_{H 2}}}
$$

Whereas the proportionality factor for the Laplace gas diffusion resistance, i.e. the pressure dependent part, can be obtained using the Fuller Schettler Giddings equation, ${ }^{19}$ which estimates the molecular diffusion coefficient for a binary gas mixture

$$
D_{A, B}=\frac{0.001 T^{1.75}}{P\left(v_{A}^{\frac{1}{3}}+v_{B}^{\frac{1}{3}}\right)^{2}} \sqrt{\frac{1}{M_{A}}+\frac{1}{M_{B}}}
$$

With the definition of the diffusion resistance

$$
R_{A, B}=\frac{l}{D_{A, B}}
$$

this yields

$$
R_{O 2, p}=R_{H 2, p} \sqrt{\frac{\frac{1}{\mathrm{M}_{\mathrm{H} 2}}+\frac{1}{\mathrm{M}_{\mathrm{Ar}}}}{\frac{1}{\mathrm{M}_{\mathrm{O} 2}}+\frac{1}{\mathrm{M}_{\mathrm{N} 2}}}}\left(\frac{\mathrm{v}_{\mathrm{O} 2}^{\frac{1}{3}}+\mathrm{v}_{\mathrm{N} 2}^{\frac{1}{3}}}{\mathrm{v}_{\mathrm{H} 2}^{\frac{1}{3}}+\mathrm{v}_{\mathrm{Ar}}^{\frac{1}{3}}}\right)^{2}
$$

In these equations $\mathrm{M}_{\mathrm{H} 2}$ and $\mathrm{M}_{\mathrm{O} 2}$ are the molar masses and $\mathrm{v}_{\mathrm{H} 2}$ and $\mathrm{v}_{\mathrm{O} 2}$ are the Diffusion volumes of hydrogen and oxygen and 1 is the diffusion distance.

The cell MTR can be simplified into a series connection of MTRs of the GDL $R_{O 2, G D L}$, of the MPL $R_{O 2, M P L}$, of the CL pores $R_{O 2, C L}$ and of the ionomer covering the active catalyst surface $\frac{1}{R F} R_{O 2, \text { Local cat }}$ (Figure 1).
The pressure-independent portion of these resistances $R_{O 2, p \text { indep }}$ corresponds to the Knudsen resistance (particle collision with pore walls) of the MPL $R_{O 2, M P L, K n u d s e n}$ and of the CL $R_{O 2, C L, K n u d s e n}$ and the local MTR $\frac{1}{R F} R_{O 2, \text { Local, cat }}$.

$$
R_{O 2, p \text { indep }}=R_{O 2, M P L, \text { Knudsen }}+R_{O 2, C L, \text { Knudsen }}+\frac{1}{R F} R_{O 2, \text { local }, \text { cat }}
$$

The local MTR comprises the resistance of the ionomer layer and its interfaces (gas-ionomer and ionomer-Pt). Since the local MTR occurs at or near the catalyst surface it is usually related to the electrochemically active surface area (ECSA) of the Pt catalyst. ${ }^{23}$ In order to relate it to the geometric cell area, it is divided by the roughness factor, i.e. the ratio of ECSA and geometric cell area. Hence, it scales in a way that is inversely proportional to the roughness factor RF and thereby the electrochemically active surface area. In the following, the local MTR related to the geometric cell area is expressed as:

$$
R_{O 2, \text { Local }, \text { geo }}=\frac{1}{R F} R_{O 2, \text { local }, \text { at }}
$$

The pressure dependent resistance $R_{O 2, p}$ corresponds to the Laplace diffusion resistance (inter particle collision) of the GDL, the MPL $\left(R_{O 2, M P L, L a p l a c e}\right)$ and the CL $\left(R_{O 2, C L, \text { Laplace }}\right)$.

$$
R_{O 2, p}=R_{O 2, G D L}+R_{O 2, M P L, \text { Laplace }}+R_{O 2, C L, \text { Laplace }}
$$

In order to investigate if an increased water uptake in the CL pore space is contributing to the increased MTR of the aged cells, the total cell resistance was additionally calculated from the limiting current in the $\mathrm{H}_{2}$ /Air configuration. The total mass transport resistance in the $\mathrm{H}_{2}$ /air configuration $R_{02, p o l}$, i.e. including liquid water in pore space, is obtained by

$$
R_{02, p o l}=\frac{4 F C_{O 2, \text { channel }}}{\mathrm{i}_{\text {lim }}}
$$

where $C_{O 2, \text { channel }}$ is the mean oxygen concentration in the channel, considering humidity, oxygen consumption and water production. It should be noted that the limiting current in $\mathrm{H}_{2}$ /air is affected by ohmic resistance and kinetic overpotentials. Further, at limiting current, more liquid water is produced and occupies the pore space, than in regular operation at lower current densities. Both lead to an overestimation of the MTR. However, since ohmic resistance and kinetic overpotentials are only marginally increasing over the AST cycles, measuring the mass transport resistance by limiting current in $\mathrm{H}_{2}$ /air allows a good estimation of the MTR increase caused by a change in water management.

\section{Results and Discussion}

Polarization measurements.-Figure 2a shows the polarization curves of the pristine cell and after 100, 500 and 1000 AST cycles. The DOE target of a maximum loss in performance of $30 \mathrm{mV}$ at 1.5 A cm ${ }^{-1}$ compared to the pristine cell, is slightly exceeded after 500 cycles and, with a value of $141 \mathrm{mV}$, significantly exceeded after 1000 cycles. Since this work is focused on the range of degradation, relevant for PEMFC vehicle operation, samples were not aged further.

Figure $2 b$ displays the contributions of the individual overpotentials. At the reference conditions, the kinetic and ohmic overpotentials increase only slightly, by $20 \mathrm{mV}$ and $10 \mathrm{mV}$ respectively, while the sum of MTR and protonic CL resistance overpotential increases by $111 \mathrm{mV}$ over the ageing cycles. The increase of protonic transport resistance is assumed to be negligible due to highly humidified conditions as found by Jomori et al. ${ }^{24}$ Thus, with approx. $80 \%$ of the total loss the MTR overpotential accounts for the major part of the electrochemical performance loss. For this reason, the present work is focused on investigating the increase in the MTR related to carbon corrosion.

Microstructure and transport parameters.-From each investigated ageing state (pristine, 100, 500 and 1000 AST cycles) a volume of $3 \mu \mathrm{m} \times 3.9 \mu \mathrm{m} \times 1.2 \mu \mathrm{m}$ of the cathode catalyst was reconstructed 

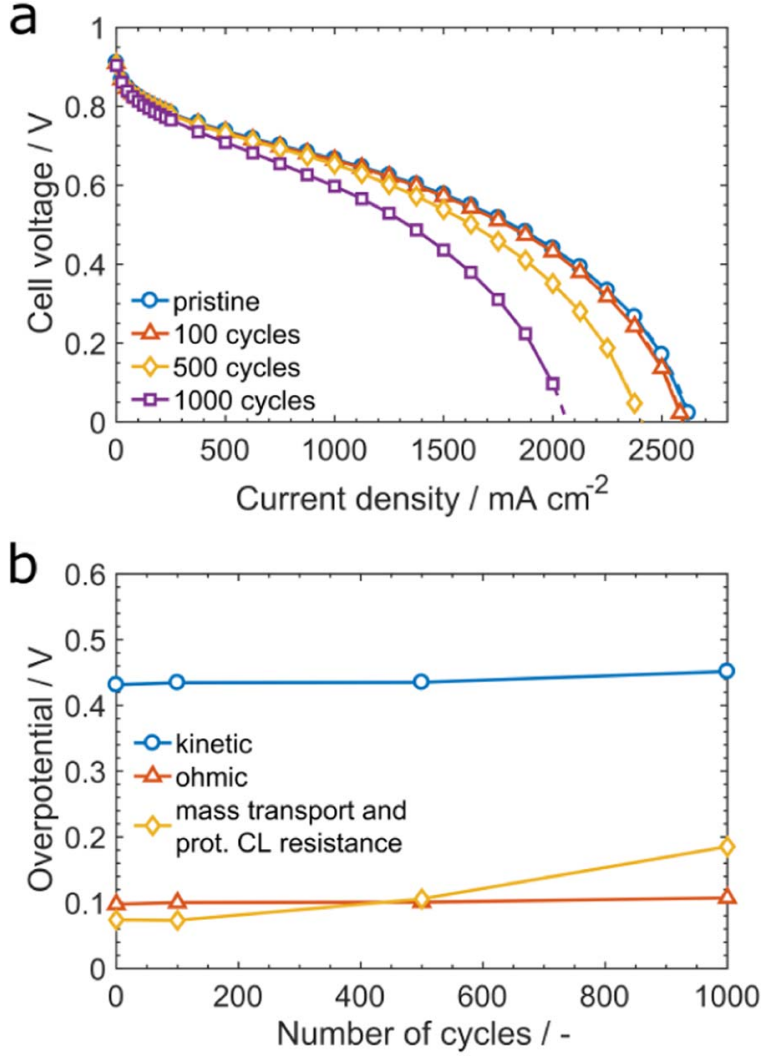

Figure 2. Polarization curves of the pristine cell and after 100, 500 and 1000 AST cycles measured at $80^{\circ} \mathrm{C}, 80 \% \mathrm{RH}$ and $1.5 \mathrm{bar}_{\text {abs }}$ (a). Overpotentials at $1.5 \mathrm{~A} / \mathrm{cm}^{2}$ calculated from HFR measurement and Tafel fit of the HFR free voltage vs. number of AST cycles (b). Linearly interpolated lines between measuring points serve as a guide to the eye. via FIB-SEM tomography (Figure 3). Infiltration of $\mathrm{ZnO}$ via atomic layer deposition enabled discrimination between pores and solid material. The carbon support, the platinum catalyst and the ionomer phase lack contrast between each other. Hence, all the three solid materials were reconstructed as a single material phase (bright gray in the figure). Within the reconstructed cathode microstructures, porosities and diffusivities of the different ageing states were to investigate the impact of CL pore space on the increased MTR.

As depicted in Figure 4a, the porosity only changes marginally over the investigated AST cycle range. First, it increases due to hollowing of the porous carbon support as the carbon starts to corrode, and subsequently decreases due to a partial collapse of the weakened carbon support, as found by Fuller et al. ${ }^{7}$ At the same time, the thickness decreases first slowly, and then more rapidly, as the support structure starts to collapse. The loss of carbon can be quantified by the equivalent solid thickness, which is the solid volume per geometric cell area. As shown in the figure, a slightly larger decrease of solid thickness is seen at the beginning of stress testing followed by a minor decrease after 500 AST cycles, corresponding to an average solid phase loss rate of $-0.5 \mathrm{~nm} /$ cycle.

As shown in Figure $4 b$ the diffusivity of the open pore space, i.e. without liquid water in the pore space, follows the trend of porosity. Since the change in diffusivity of the CL pore space is only minor, it is not considered to have a significant impact on the performance loss. This finding is counterintuitive and in contrast to common understanding. It is therefore investigated further in the following section.

Mass transport resistance.-Measuring the limiting current in the hydrogen pump setup, as described in Electrochemical measurements section, allows quantification of the oxygen MTR without the effect of liquid water in the pore space and local heat generation. By varying the pressure, the MTR can be separated into a pressure dependent part and a pressure independent part of the MTR. As shown in Figure 5, the pressure dependent MTR, represented by the slope of the total MTR over the absolute pressure, only changes slightly in the tested ageing range. Hence, the major part of the MTR increase (approx. 90\% at
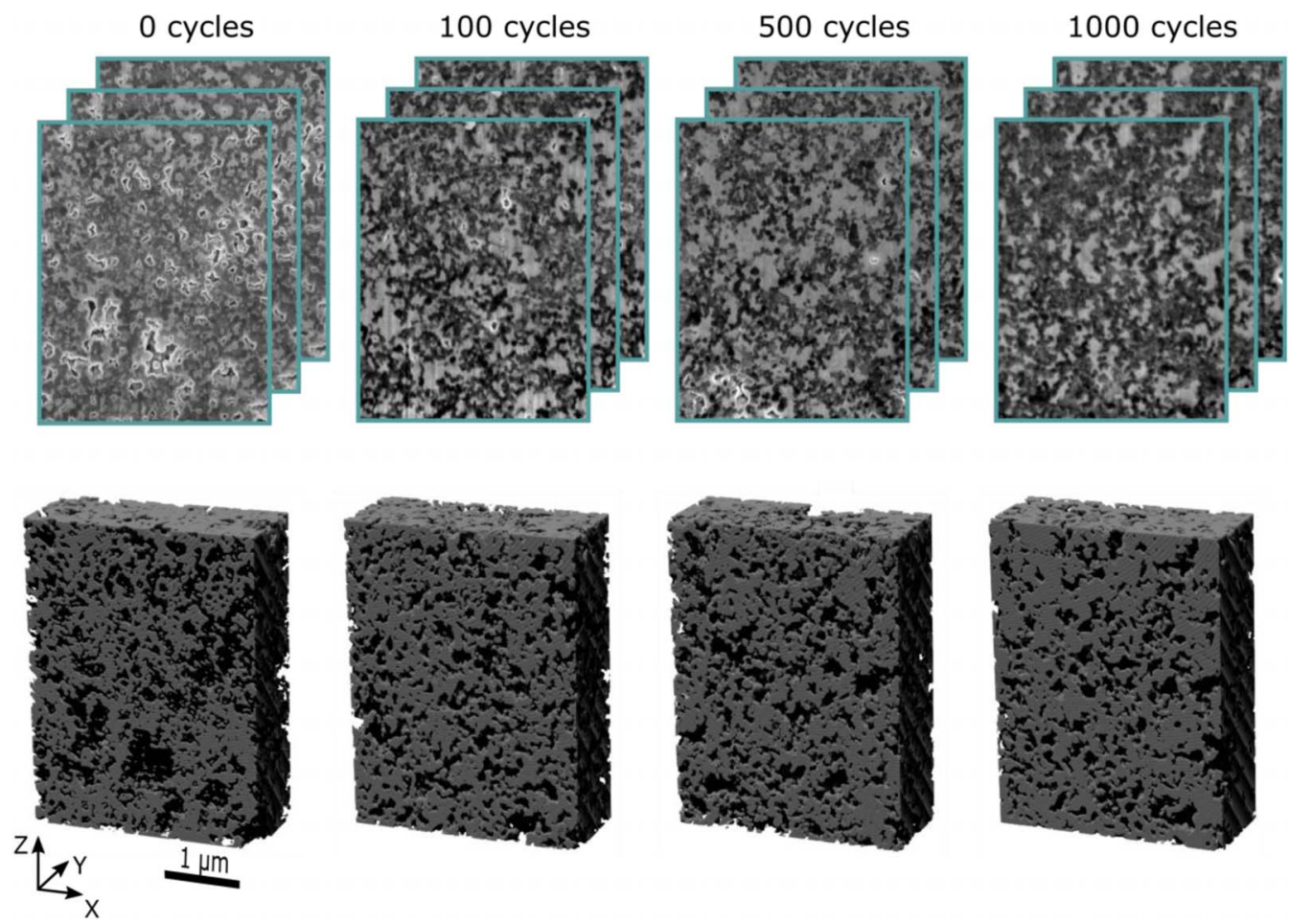

Figure 3. Raw images and 3-D representation of the reconstructed volume $(3 \mu \mathrm{m} \times 3.9 \mu \mathrm{m} \times 1.2 \mu \mathrm{m})$ of the cathode of the pristine sample, after 100,500 and 1000 AST cycles. The pores were infiltrated with $\mathrm{ZnO}$ (bright gray) to increase the contrast to the solid phase (dark gray). The solid phase comprises the carbon support, the platinum catalyst and the ionomer phase. 

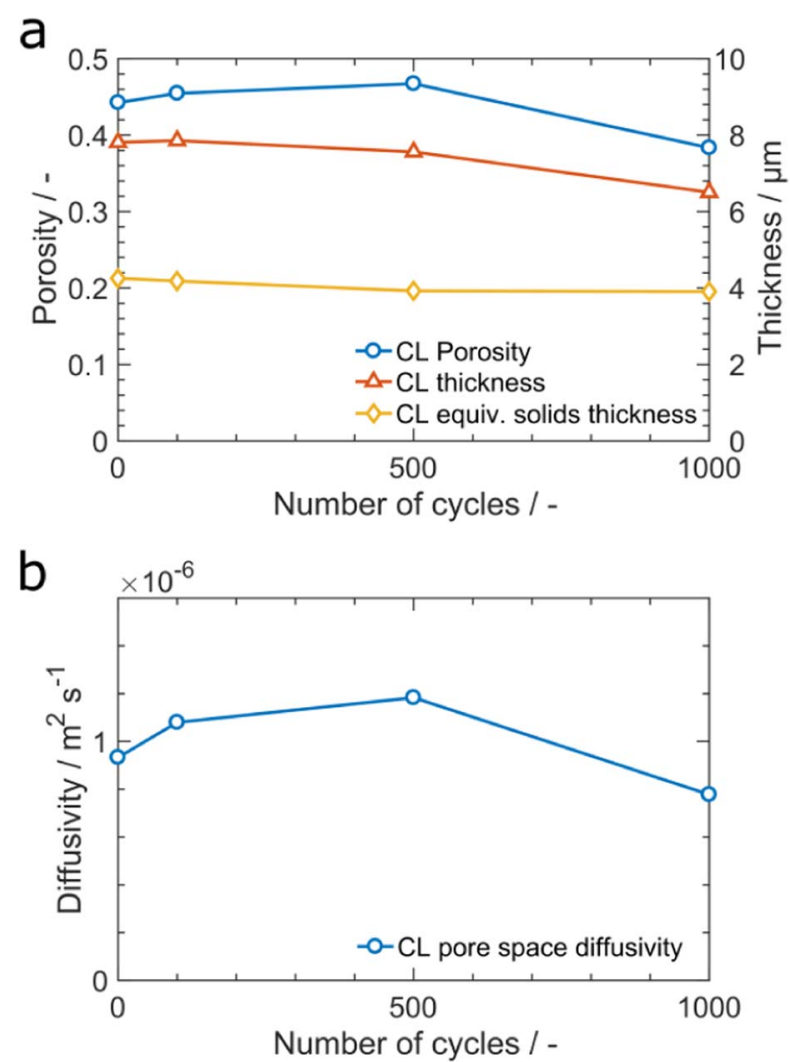

Figure 4. Porosity, overall thickness equivalent solids thickness (a) and Bosanquet diffusivity at $80^{\circ} \mathrm{C}, 1.5 \mathrm{bar}_{\text {abs }}$ (b) of the cathode catalyst layer vs. number of AST cycles. The median pore diameter $\mathrm{D}_{50}$ is between $77 \mu \mathrm{m}$ and $81 \mu \mathrm{m}$ as determined by FIB-SEM tomography.

reference conditions) is due to an increase of the pressure independent MTR $R_{O 2, p \text { indep }}$, corresponding to the Y-axis intercept.

Figure 6a shows the pressure independent and pressure dependent MTR of the cell (GDL, MPL and CL) and of the CL pore space vs. the number of AST cycles at $80^{\circ} \mathrm{C}, 1.5 \mathrm{bar}_{\mathrm{abs}}, 80 \% \mathrm{RH}$. The cell mass transport resistance was determined by hydrogen pumping and the CL mass transport resistance was calculated from the pore space diffusivity and the thickness of the CL, as determined by FIB/SEM tomography. The pore space diffusivity comprises the Laplace diffusivity yielding the pressure dependent part and the Knudsen diffusivity yielding the pressure independent part of the MTR in the CL pore space (Theory section). The additional MTR due to liquid water in the pore

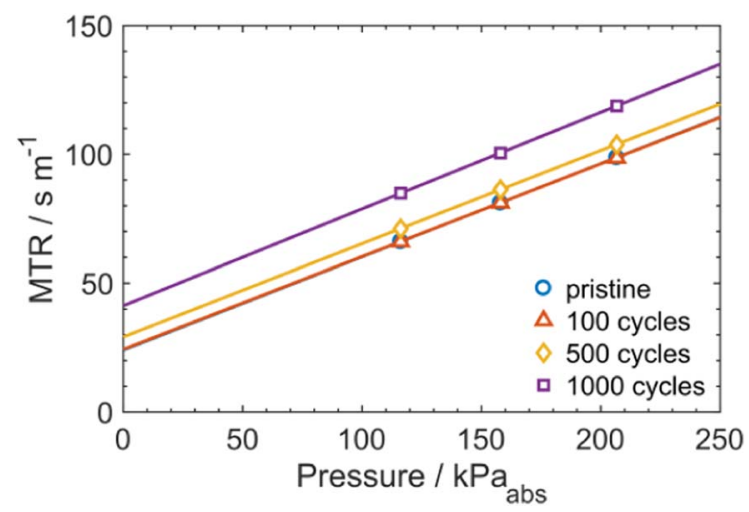

Figure 5. Total mass transport resistance for the pristine cell, after 100, 500 and 1000 AST cycles vs. absolute cell pressure, determined by limiting current measurements in a hydrogen pump setup.

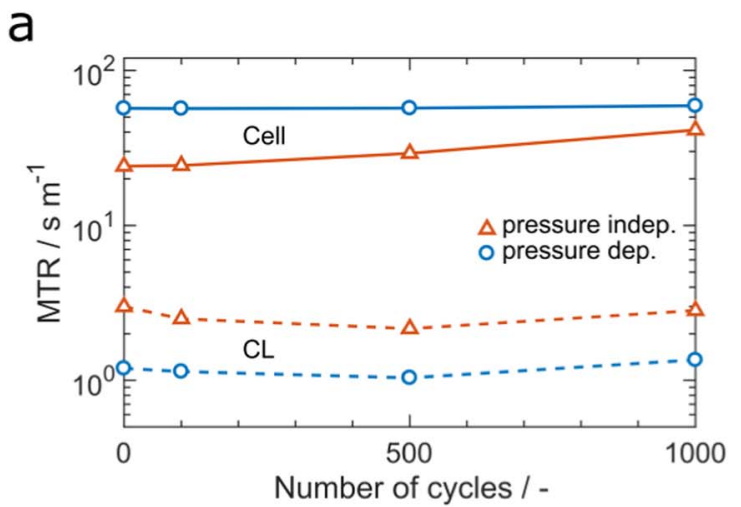

b
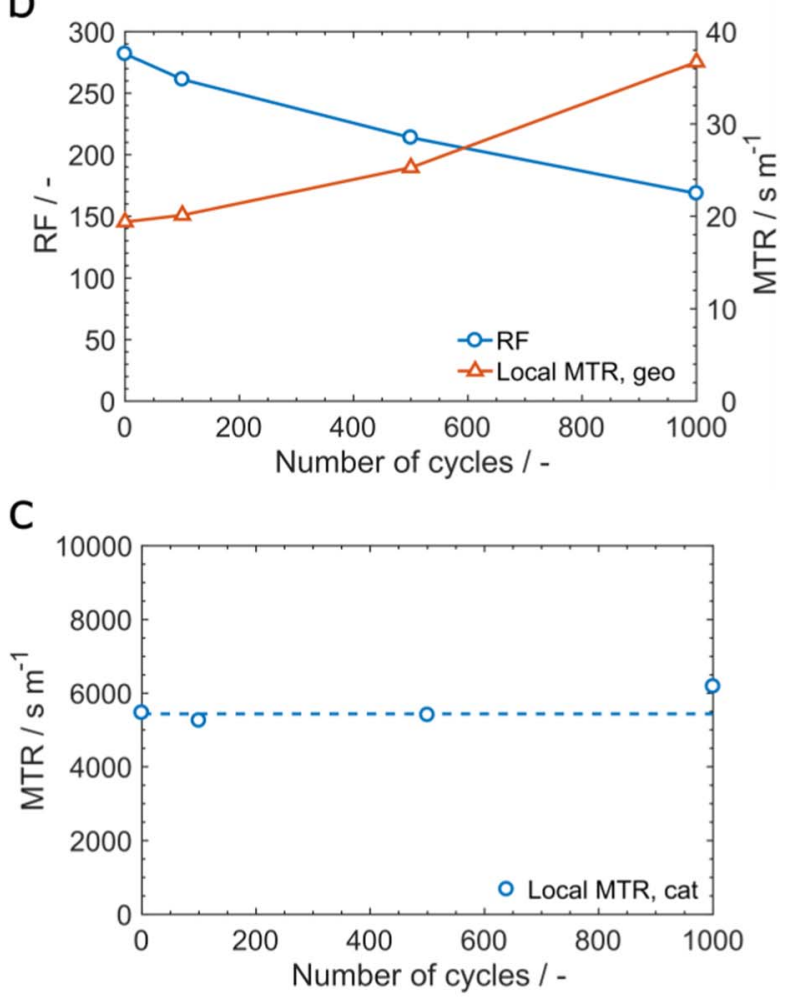

Figure 6. a) Pressure independent and pressure dependent mass transport resistances of the cell (GDL, MPL and CL), as determined by hydrogen pumping, and of the CL pore space, determined by tomography, vs. number of AST cycles. b) Local mass transport resistance (related to the geometric cell area), as determined by hydrogen pumping and roughness factor vs. ageing cycles. c) Local mass transport resistance at active catalyst surface vs. number of AST cycles. Operating conditions: $80^{\circ} \mathrm{C}, 1.5 \mathrm{bar}_{\mathrm{abs}}, 80 \% \mathrm{RH}$.

space is excluded by using the hydrogen pump method and calculating the diffusivities of the dry pore space. While the pressure independent part of the cell MTR (i.e. Knudsen resistance in pores and local resistance at the catalyst, see Theory section) increases from $24.1 \mathrm{sm}^{-1}$ to $41.3 \mathrm{sm}^{-1}$, the MTR of the CL pore space slightly decreases and then increases back to its original value. However, the CL pore MTR is relatively small ( 3 to $4 \mathrm{sm}^{-1}$ ), when no liquid water is involved, as also found by Nonoyama et al. ${ }^{25}$ As a consequence, the CL pore space MTR is negligible in the dry case and only the local MTR has a significant impact on the increase of the cell MTR in the series resistance model (Theory section).

Assuming a constant value for $R_{O_{2}, M P L, \text { Knudsen }}=1.75 \mathrm{~s} / \mathrm{m}^{26}$ and considering $R_{O_{2}, C L, \text { Knudsen }}$ from the tomography, the cell area-related local MTR $\left(R_{O 2, \text { local,geo }}\right)$ can be calculated (see theory, Equations 14 and 15). As shown in Figure 6b, the local transport resistance increases 


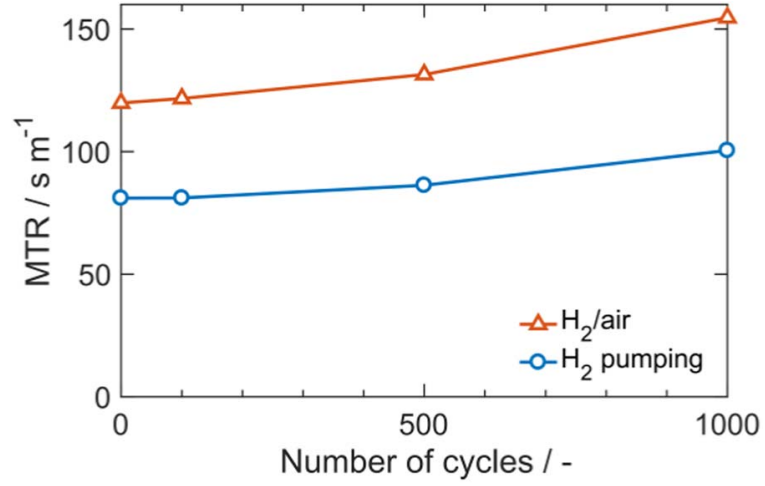

Figure 7. Cell mass transport resistance vs. number of AST cycles, determined by limiting current measurements in the hydrogen pump setup and in the air/hydrogen setup at $80^{\circ} \mathrm{C}, 1.5 \mathrm{bar}_{\mathrm{abs}}, 80 \% \mathrm{RH}$.

with the ageing cycles by $17.3 \mathrm{~s} / \mathrm{m}$ over 1000 cycles. At the same time the roughness factor, i.e. the ratio of electrochemically active Pt surface area to geometric cell area, decreases in a similar fashion as the local MTR increases. Taking the decreasing roughness factor into account yields the active surface area related local MTR $\left(R_{O_{2}, \text { local }, \text { cat }}\right)$. The values of approx. 5000 to $6000 \mathrm{~s} \mathrm{~m}^{-1}$ are relatively large, ${ }^{18}$ but still in the range of values found in literature. ${ }^{25,27}$ As Figure $6 \mathrm{c}$ shows, $R_{O_{2}, \text { local cat }}$ is approx. constant up to 500 AST cycles. After 1000 cycles the local MTR has increased by $13 \%$. This could either be explained by an actual increase of the local MTR, e.g. due to a change of the carbon structure within the agglomerates, which is not detected since it is below the FIB-SEM resolution. Alternatively, $R_{O_{2}, \text { local,cat }}$ remains constant and the pressure independent resistance increases due to an additional Knudsen transport resistance, which is not captured by this work. This additional resistance could stem from the MPL, the CL/MPL interface or from the increasing ionomer to carbon ratio leading to an increased effect of ionomer swelling, which would not have been captured by this work. Concluding, it can be stated, that the major part of the dry MTR increase is caused by the decreased electrode roughness. This is consistent with the results of a combined experimental and modelling degradation study by Jomori et al. ${ }^{24}$ investigating the impact of active surface area loss on performance of a PEMFC after cycling between 0 and $0.9 \mathrm{~V}$. Although this potential range is typically used for Pt particle degradation and lower than the potential range used in this work, it also leads to a loss of ECSA as our protocol and has therefore a comparable effect on local MTR. ${ }^{11}$

To investigate the effect of carbon corrosion on water management in the CL, we compared the cell MTR determined from $\mathrm{H}_{2} /$ air limiting current to that of hydrogen pumping (Figure 7). As expected the MTR in $\mathrm{H}_{2}$ /air polarization is larger at every ageing state, due to liquid water in the GDL and probably in the CL pores. In addition, the increase of $34.9 \mathrm{sm}^{-1}$ (vs. $19.5 \mathrm{sm}^{-1}$ ) during the AST cycle range is higher. This additional MTR increase indicates a larger water uptake of the aged cell. It is to note, that the limiting current density of the $\mathrm{H}_{2}$ /air polarization curve was higher than the reference current density of $1.5 \mathrm{~A} \mathrm{~cm}^{-2}$, involving major water production and thus higher liquid water saturation in the pore space. For this reason, the contribution of water uptake to the MTR increase is smaller in regular operation.

Comparing the individual parts of the MTR increase after 1000 AST cycles shows, the largest part (50\%) is caused by the increase of the local MTR $\left(R_{O 2, \text { local,geo }}\right)$ mainly due to a loss of active surface area. At limiting current, $44 \%$ of the increase is due to increased water uptake and the remaining $6 \%$ are due to additional resistances e.g. in the MPL or at the CL/MPL interface which are not captured by this work.

\section{Conclusions}

In this work, the reasons for the performance loss, related to carbon support corrosion of PEMFC cathodes were investigated. MEAs were aged in an accelerated stress test by voltage cycling in a hydrogennitrogen configuration between 1 and 1.5 V. Performance degradation was captured by measuring polarization behavior in hydrogen-air operation at $80^{\circ} \mathrm{C}, 1.5$ bar $_{\text {abs }}$ and $80 \% \mathrm{RH}$. After 1000 stress test cycles the increase in overpotential was $141 \mathrm{mV}$ at $1.5 \mathrm{~A} \mathrm{~cm}^{-2}$, thus exceeding the limit given by the DOE of $30 \mathrm{mV}$ at $1.5 \mathrm{~A} \mathrm{~cm}^{-2}$. At these conditions, approx. $79 \%$ of the performance degradation of the tested cell was caused by an increased MTR. The rest was assigned to an increase of kinetic overpotential (14\%) and ohmic overpotential $(7 \%)$. In order to find the cause of the increased MTR, structural changes in the porous cathode catalyst structure were analyzed via FIB-SEM tomography and the porosity, the layer thickness and the MTR of the catalyst layer pore space were calculated. The catalyst pore space MTR was found to be small in comparison to the cell MTR ( 3 to $4 \mathrm{sm}^{-1}$ vs. 120 to $155 \mathrm{sm}^{-1}$ ), showing, that the structural changes of the CL pore space are not the main reason for the observed performance degradation. The cell MTR was determined by limiting current experiments in a hydrogen pump and in a hydrogen-air configuration, yielding the MTR with and without the influence of water generation in the catalyst layer. With the tomography and the electrochemical measurements, the individual shares of the MTR due to the respective degradation mechanisms were estimated. At limiting current in hydrogen/air, the major portion of the MTR increase (50\%) is caused by an increase of the local MTR due to a loss of active surface area. $44 \%$ are caused by an increased water uptake and the remaining $6 \%$ are due to other resistances e.g. in the MPL or at the CL/MPL interface which are not captured by this work. At conditions below limiting current, the liquid water saturation is lower, reducing the impact of the decreased hydrophobicity.

\section{Acknowledgments}

We acknowledge funding from the Fuel Cells and Hydrogen 2 Joint Undertaking (grant No. 700127) within the project INSPIRE. The Fuel Cells and Hydrogen 2 Joint Undertaking is supported by the European Union's Horizon 2020 research and innovation program, Hydrogen Europe and Hydrogen Europe Research.

\section{ORCID}

Simon Thiele (D) https://orcid.org/0000-0002-4248-2752

Severin Vierrath (1) https://orcid.org/0000-0002-4505-2803

\section{References}

1. L. Dubau, L. Castanheira, F. Maillard, M. Chatenet, O. Lottin, G. Maranzana, J. Dillet, A. Lamibrac, J.-C. Perrin, E. Moukheiber, A. ElKaddouri, G. de Moor, C. Bas, L. Flandin, and N. Caqué, WIREs Energy Environ, 3(6), 540 (2014).

2. C. A. Reiser, L. Bregoli, T. W. Patterson, J. S. Yi, J. D. Yang, M. L. Perry, and T. D. Jarvi, Electrochem. Solid-State Lett., 8(6), A273 (2005).

3. H. Schulenburg, B. Schwanitz, N. Linse, G. G. Scherer, A. Wokaun, J. Krbanjevic, R. Grothausmann, and I. Manke, The Journal of Physical Chemistry C, 115(29), $14236(2011)$

4. Y.-C. Park, K. Kakinuma, M. Uchida, D. A. Tryk, T. Kamino, H. Uchida, and M. Watanabe, Electrochim. Acta, 91, 195 (2013).

5. S. H. Lau, Wilson K S Chiu, F. Garzon, H. Chang, A. Tkachuk, M. Feser, and W. Yun, J. Phys.: Conf. Ser., 152, 12059 (2009).

6. J. Durst, A. Lamibrac, F. Charlot, J. Dillet, L. F. Castanheira, G. Maranzana, L. Dubau F. Maillard, M. Chatenet, and O. Lottin, Applied Catalysis B: Environmental, 138, 416 (2013).

7. A. G. Star and T. F. Fuller, J. Electrochem. Soc., 164(9), F901 (2017)

8. N. Macauley, D. D. Papadias, J. Fairweather, D. Spernjak, D. Langlois, R. Ahluwalia, K. L. More, R. Mukundan, and R. L. Borup, J. Electrochem. Soc., 165(6), F3148 (2018).

9. B. T. Sneed, D. A. Cullen, K. S. Reeves, O. E. Dyck, D. A. Langlois, R. Mukundan, R. L. Borup, and K. L. More, ACS Applied Materials \& Interfaces, 9(35), 29839 (2017).

10. J. K. Hyun, Z. Y. Liu, and D. A. Muller, Microsc Microanal, 15(S2), 1418 (2009).

11. USDRIVE, Fuel Cell Technical Team Roadmap, 2017

12. S. Vierrath, F. Güder, A. Menzel, M. Hagner, R. Zengerle, M. Zacharias, and S. Thiele, J. Power Sources, 285, 413 (2015).

13. J. Becker, C. Wieser, S. Fell, and K. Steiner, International Journal of Heat and Mass Transfer, 54(7-8), 1360 (2011). 
14. CRC Press, Editor, CRC Handbook of Chemistry and Physics: 81st ed. (2000-2001).

15. M. Suermann, K. Takanohashi, A. Lamibrac, T. J. Schmidt, and F. N. Büchi, J. Electrochem. Soc., 164(9), F973 (2017).

16. J. Maier, Angew. Chem., 102(7), 847 (1990).

17. D. R. Baker, D. A. Caulk, K. C. Neyerlin, and M. W. Murphy, J. Electrochem. Soc., 156(9), B991 (2009).

18. Thomas A. Greszler, David Caulk and and Puneet Sinha, J. Electrochem. Soc. 159(12), F831 (2012).

19. F. B. Spingler, A. Phillips, T. Schuler, M. C. Tucker, and A. Z. Weber, International Journal of Hydrogen Energy, 42(19), 13960 (2017).

20. A. T. S. Freiberg, M. C. Tucker, and A. Z. Weber, Electrochemistry Communications, 79, 14 (2017).
21. T. Schuler, A. Chowdhury, A. T. S. Freiberg, B. Sneed, F. B. Spingler, M. C. Tucker, K. L. More, Clayton J. Radke, and Adam Z. Weber, J. Electrochem. Soc., 166(9) (2019).

22. R. O'hayre, S.-W. Cha, F. B. Prinz, and W. Colella, Fuel cell fundamentals, John Wiley \& Sons (2016).

23. Jon P. Owejan, Jeanette E. Owejan, and Wenbin Gua, J. Electrochem. Soc., 160(8), F824 (2013)

24. S. Jomori, N. Nonoyama, and T. Yoshida, Journal of Power Sources, 215, 18 (2012).

25. Nobuaki Nonoyama, Shinobu Okazaki, and Z Adam, Weber, Yoshihiro Ikogi, Toshihiko Yoshida, J. Electrochem. Soc., 158(4), B416 (2011).

26. L. M. Pant, S. K. Mitra, and M. Secanell, Journal of Power Sources, 206, 153 (2012).

27. K. Kudo, T. Suzuki, and Y. Morimoto, p. 1495, ECS (2010). 